\title{
Inspection of Bottles Crates in the Beer Industry through Computer Vision
}

\author{
Mário Campos**, Manuel Ferreira*, Teresa Martins**, Cristina Santos* \\ *Department of Industrial Electronics \\ University of Minho \\ Campus de Azurem - Guimarães, Portugal \\ Telf: +351253510190 ; fax: +351253510189 ; \\ email: mjf@dei.uminho.pt,cristina@dei.uminho.pt \\ **Enermeter, Sistemas de Medição, SA \\ Praceta das Agras, 26 \\ Celeiros - Braga, Portugal \\ Telf: +351253287237 ; fax: +351253287238 ; \\ email: mario_campos@portugalmail.pt,tmartins@enermeter.pt
}

\begin{abstract}
This article presents a system developed for the industry of bottling beer. The system has to perform the inspection of various items in the final stage, meaning after the production phase where the bottles are already in the crate. The items to inspect are the following: whether the crate is correct (with the correct color), whether the crate is broken, whether the crate is fully populated, i.e., all bottles are present, to check for bottles without caps and whether the capsule is the correct one. The work uses techniques of computer vision for these verifications and also principal components analysis (PCAs) for the recognition of the capsules. This system is currently installed in the assembly line and the results indicate high efficiency and confidence in the obtained solution.
\end{abstract}

\section{Introduction}

The recent technological development with respect to systems for image acquisition and data processing has led to the specification and implementation of new solutions for machine vision in various industrial fields [1,2,3]. It is the case for the filling of bottles, in which the traditional systems only concern the control of the level. Currently there has been the use of vision solutions in other subsectors of this industry, such as packaging the final product, washing the raw material, selection of products, among others. This is the case of the present solution, which intents to be applied after the filling of beer bottles that are placed in crates. Prior to these crates are sent to the final customer it is necessary to inspect several items, including: if the crate has the right color, if the crate is damaged on the handles, if all the positions of the crate have bottle, if the bottle has a capsule and if the capsule is the correct one. For each of these inspection issues different image processing and analysis algorithms have been developed, using color and gray levels images. The system is currently installed and already integrated into the production line, and in testing for final adjustments.

Figure 1 presents a top image of a specific kind of crate (yellow crate) for beer. It identifies the areas of inspection

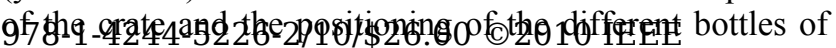
beer. Figure 2 shows an empty crate in order to illustrate the lack of bottle for each of the positions of the crates. Figure 3 and 4 present, respectively, an image of a broken crate and a crate with some bottles exchanged, i.e., with different capsules

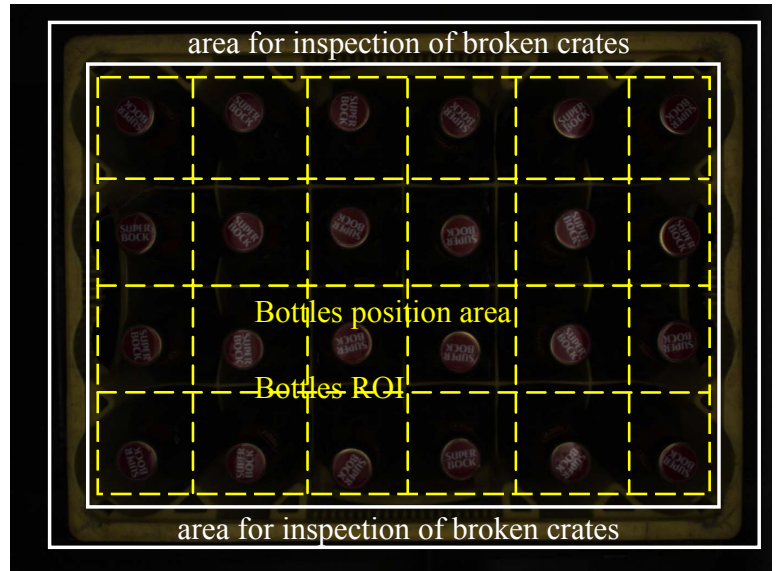

Fig. 1. Top image of a yellow crate. In white is specified the predefined area for the inspection of defects on the crate and to check the color; in yellow are defined the regions of interest (ROIs) for the segmentation of the bottles and the capsules.

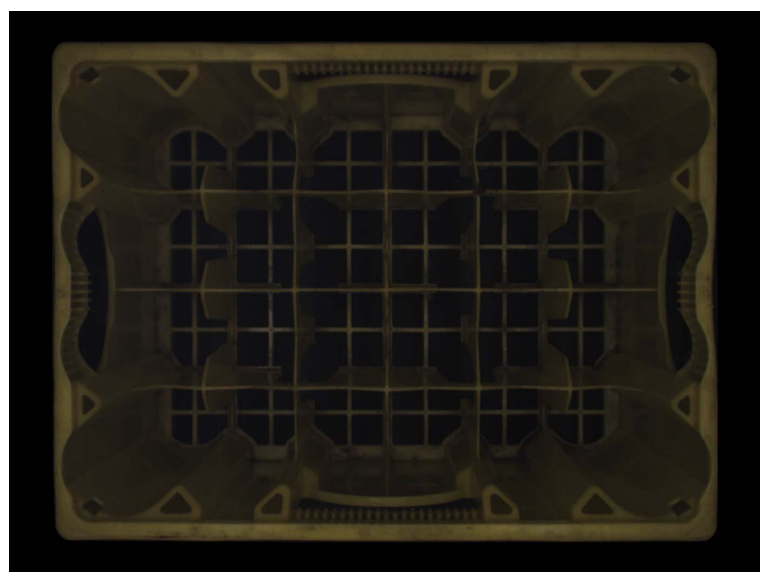

Fig. 2. Image of a yellow crate. The intention of this figure is to illustrate the content of an image without the bottles. 


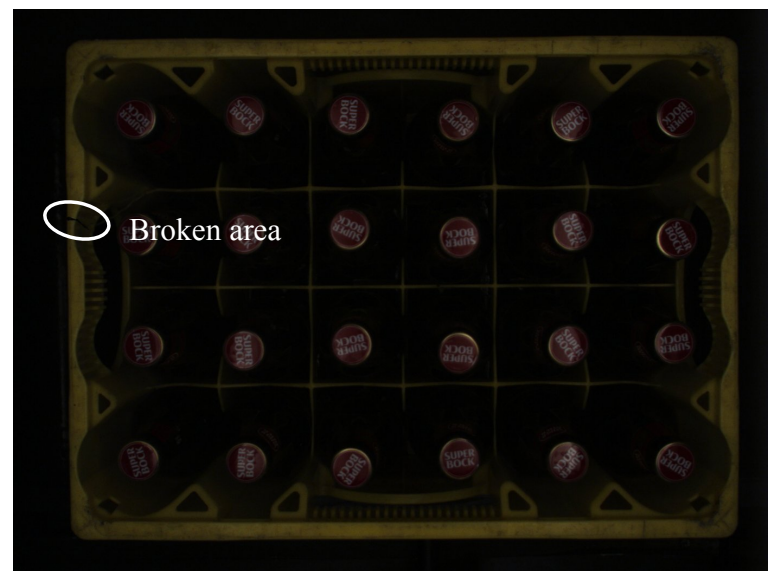

Fig. 3. Image of a broken crate.

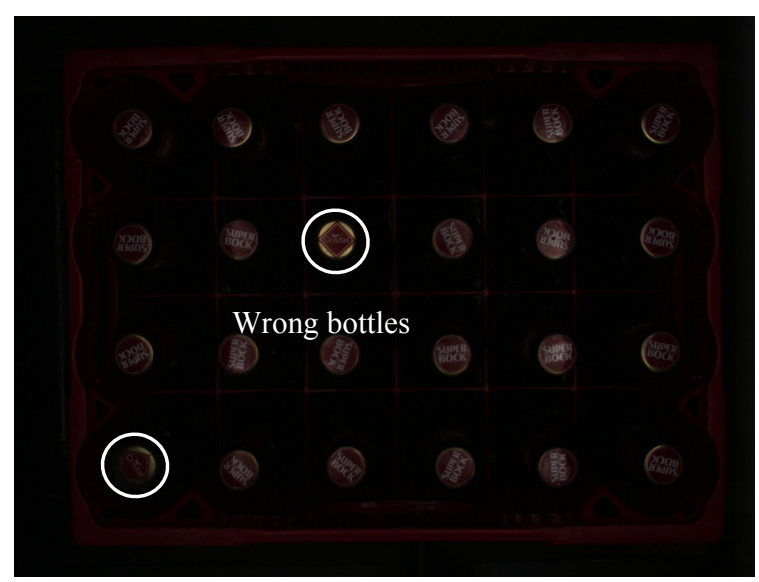

Fig. 4. Crate Image with two bottles exchanged.

\section{Methods}

Figure 5 shows, in schematic way, the setup of the vision system. It consists of a light box with fluorescent lamps of high frequency, positioned above the grade of beer. The camera, AVT Marlin F145C2 (color camera with $1392 \times 1038$ pixels resolution), is placed in a central position on the light box at the same height as this.
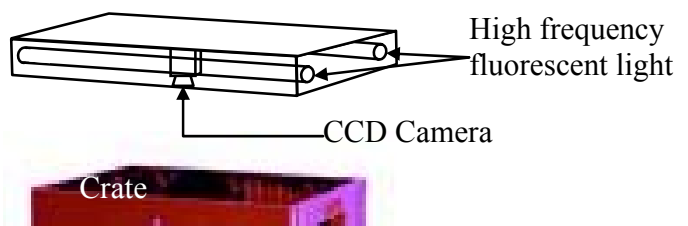

Fig. 5. Illustration of the position of lighting and camera relatively to the crate.

One of the primary requirements of the computer vision system is that it had to adapt to clear space in the line. This requirement has affected the layout of the vision system and the specifications and developments of several algorithms. The direct result of this requirement is the small size of the light box and the consequent low uniformity of illumination, which ideally was intended to be uniformly and diffuse. Another consequence concerns 11 the distance of the crate to the camera, which also had to be reduced. This implies the use of a lens with a small focal length, creating a strong perspective effect in the image.

Figure 6 presents the image of two bottles, one positioned in the center and another on the limits of the crate. These two images show the effects resulted from the perspective and lack of light uniformity.

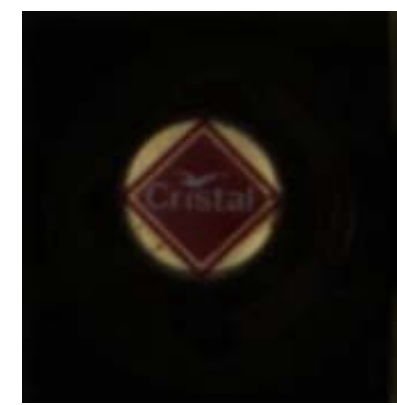

central capsule

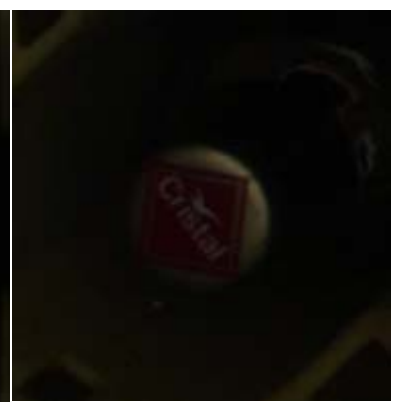

edge capsule
Fig. 6. Representative images of the differences between the capsules acquired on the center and on the periphery of the crate.

For the inspection of each item specific algorithms were developed. They were conditioned by the setup of the image acquisition system. In particular they must be able to support the poor uniformity of illumination and the strong effect of perspective. Another key requirement is that the system must support different orientations of the bottle in the crate. Thus the algorithm of recognition of the capsule has to deal with different orientations as shown in Figure 7.

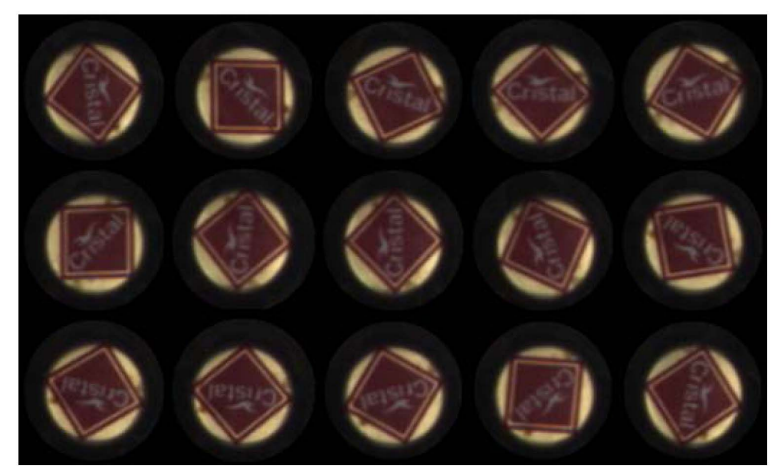

Fig. 7. Representative images of the differences between the capsules of the same crate position, acquired in different crates.

The application was developed in $\mathrm{C}++$ for the Windows operating system, using the library of image processing and analysis OpenCV [4].

The block diagram shown in Figure 8 intends to describe the different steps that must be traversed for a crate to be given as appropriate and follow in the production line. In the following sub sections each of these blocks is specified.

\section{A. Check Crate}

As stated above, owing to the imposed small size of the box lighting, the crate is not evenly illuminated. In this sense, the first step of the verification of defects in the crate (figure 9) consists in simulate a uniform illumination. 3 Phis procedure is a color mapping, dependent on the 
position of the pixel in consideration $[1,5]$. An image of a white target was acquired to reflect the non-uniformity of the illumination. The mapping of differences between the target strength and a uniform level (255) was created. An image with more uniform tone is obtained by adding this difference (mapping) to the acquired image.

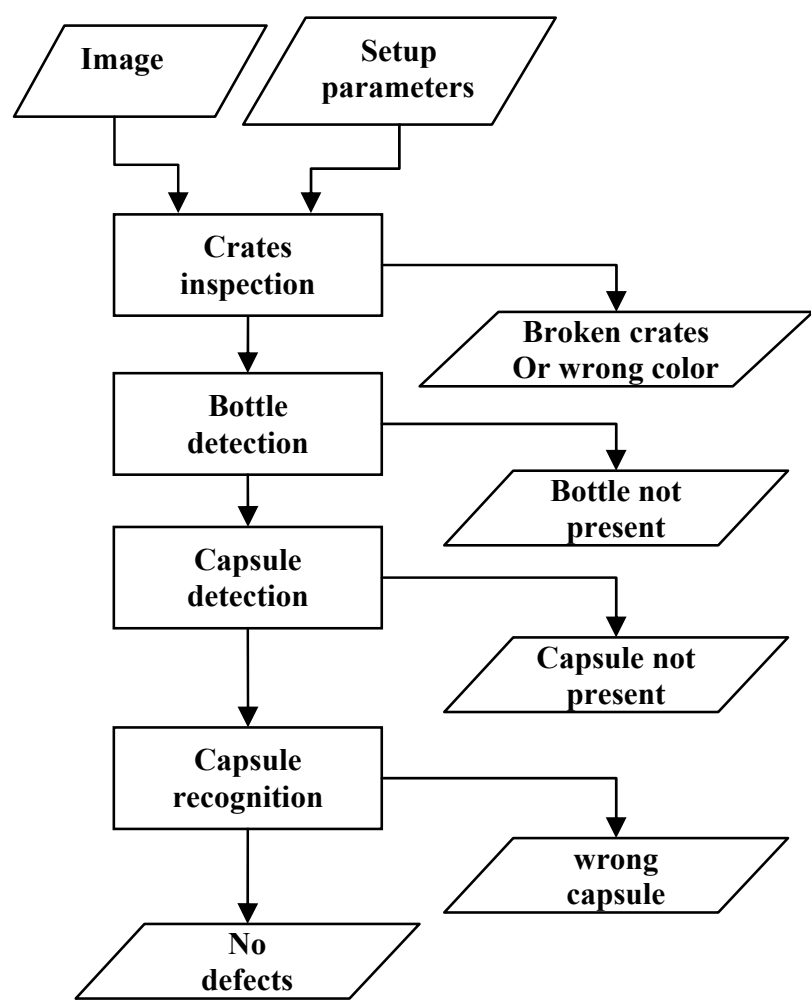

Fig. 8. General algorithm application.

Specifically, the RGB image acquired by the camera is converted to HSL $[1,2,6]$ color space. The procedure for illumination calibration is applied to the component $\mathrm{L}$. The HSL image is then converted back to RGB color space. In the resulting image, segmentation procedure with RGB thresholds specified, for the type of crate to be analyzed, is applied. In the binary image it is made a count of pixels. In the case of the resulting number of pixels (area) is less than a pre-defined threshold, then an exchange of crate exists and the global analysis procedure is stopped. In the opposite case it is determined the convex hull [1,5] of the blob of the crate. This process aims to determine the orientation of the crate compared to the axes of the image and the positioning relatively to the center of the image. This is necessary for further analysis with respect to the detection of the bottle and the capsule. After the alignment of the crate, it is quantified the distance between the contour generated by the convex hull and the contour of the blob given by the chain code. Thus, it is possible to infer broken areas of the crate, since in these situations the distance between the convex hull and contour exceeds a pre-defined threshold.

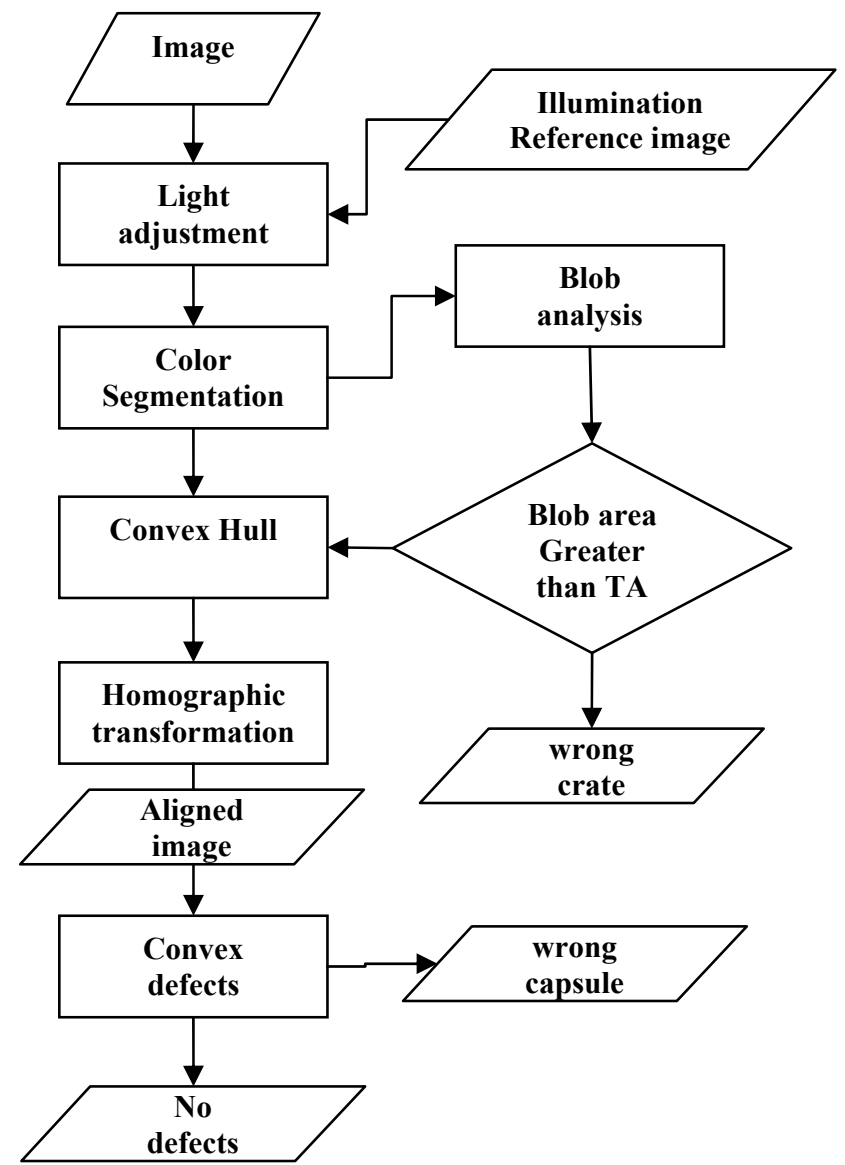

Fig. 9. Algorithm for the verification of the crate color and structure.

\section{B. Presence of the bottle}

This procedure, shown in Figure 10, is applied in the RED channel of the image aligned with the crate, i.e. crate image oriented with the axes of the image and with the center centered in the image center. In this procedure is also used a previously acquired image of an empty crate. In both images of the crate, a morphological filter is applied. After that, the resulted image of the empty crate is subtracted by the resulted image of the current crate. The morphological filter applied to the image of reference is a set of 15 dilatations, with a circular structuring element of size $3 \times 3$. The filter applied to the current image is the application of a close-up of 7 iterations using a structuring element of $3 \times 3$ circular. The application of these filters allows minimizing background details of the grid and the bottle. After subtraction, and for each region of interest that defines the position of each capsule, it is made a binarization and the area of the resulting blob is quantified. If this area exceeds a pre-defined threshold then a bottle is present. This procedure is performed for all ROIs defined in Figure 1. 


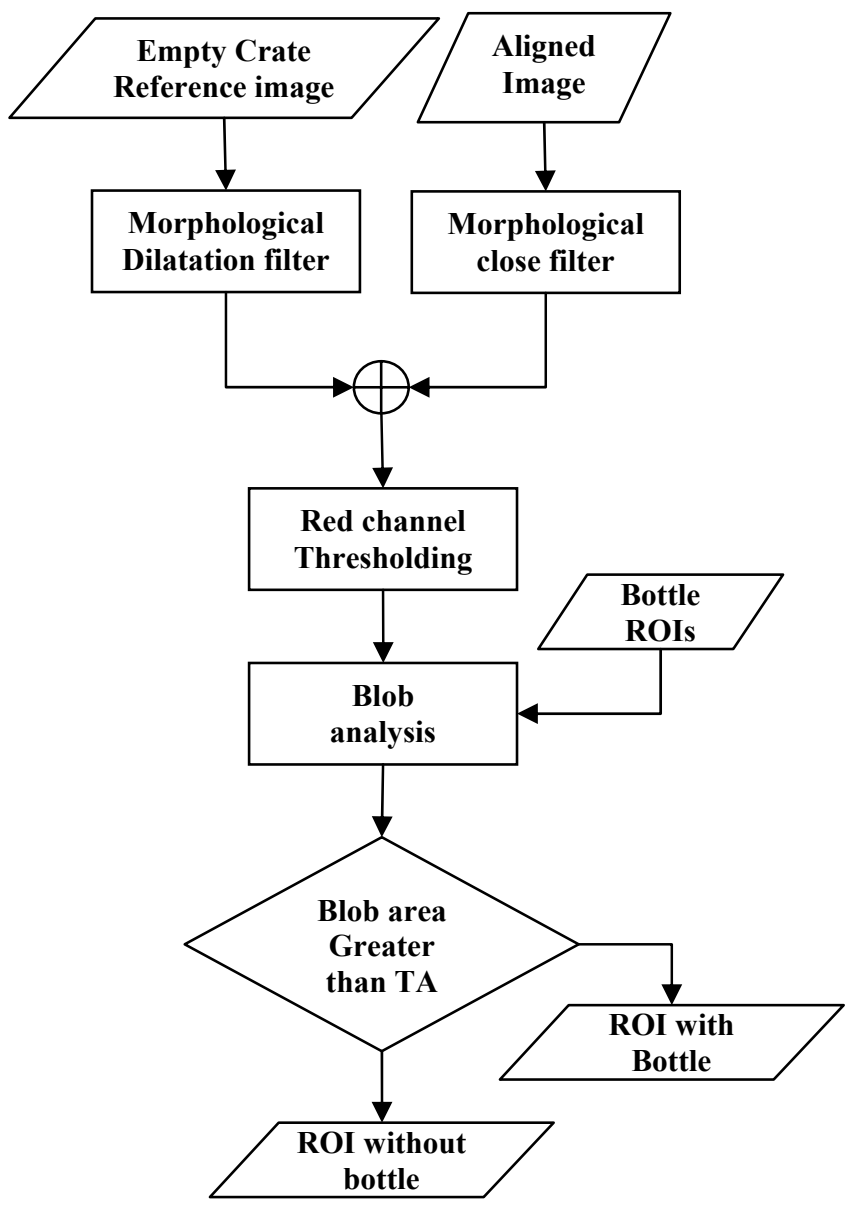

Fig. 10. Algorithm to detect the presence of bottle.

\section{Capsule segmentation}

The procedure of figure 11 allows verifying if the bottle has capsule and if so, to set a new adjust ROI, containing the capsule, to be used in identification via Principal Components Analysis (PCAs) [7] (algorithm of Figure 12). This procedure is performed on the aligned image of the crate for each ROI of the image of Figure 1.

This block is based on the application of the Canny [8] detector to the image. For the resulting contours it verifies which ones are most similar to the capsule in size and shape. Before applying the Canny detector, a close morphological filter with $3 \times 3$ structuring element is applied to the image, to minimize potential false contours. For each contour, the area under the contour and the perimeter/area ratio are quantified. If both these values are within the pre-established parameters it is considered that a capsule exists and is defined a new region of interest adjusted to the size of that contour.

\section{Capsule recognition}

After verifying the existence of the capsule in the bottle, it is necessary to determine whether that capsule is the correct one. The recognition of the capsules uses a classification process based on principal components analysis (PCAs) [9-11].
For each type of bottle and for each crate position, it was acquired a set of images of capsules that serve as a model for the PCAs.

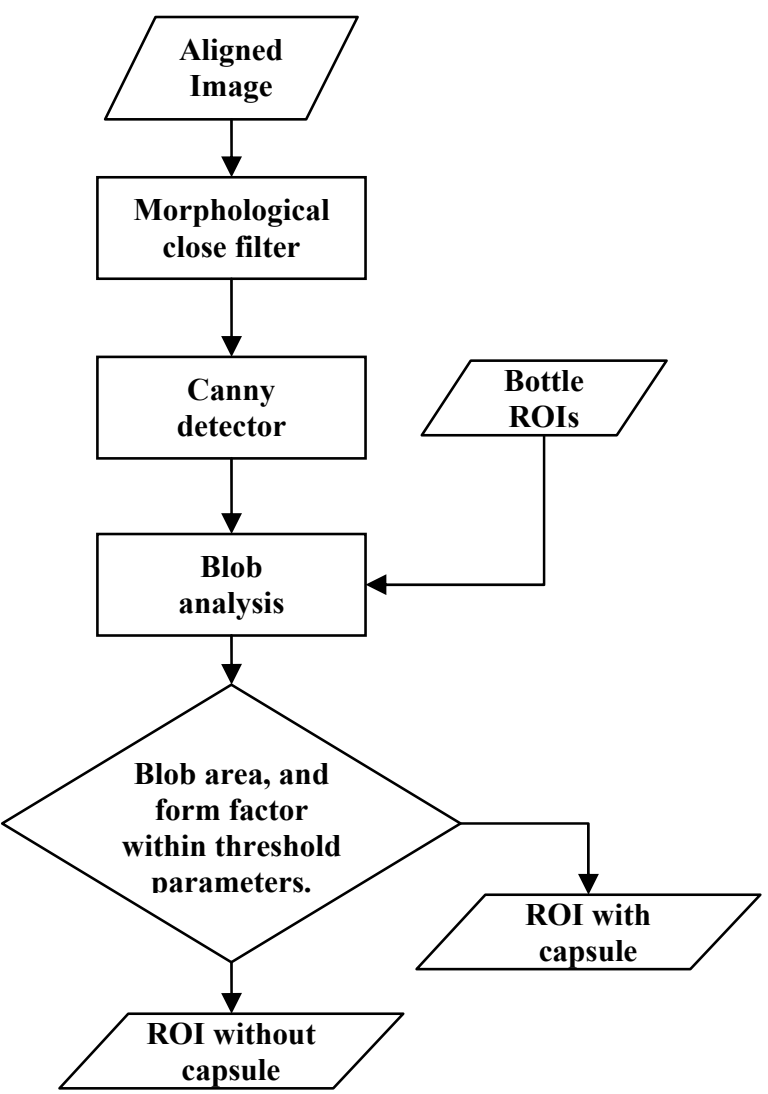

Fig. 11. Algorithm for the segmentation of the capsule.

Among the wide range of tools available, a technique that is distinguished by its great potential for pattern recognition is the principal components analysis. The PCA involves a statistical analysis of correlation between components that form the object, treated as vector structures. Through a projection of those structures in subspace vectors, a reduction in the dimensionality of the sample is obtained, and also a more meaningful representation. The PCA technique operates through the following steps:

- Data collection or sampling, in this project monochrome images of capsules - $n$ images.

- Normalization of the sample by subtracting the mean of the sample - $S N$.

- Calculation of the correlation matrix given by:

$$
\begin{aligned}
& M=(\operatorname{cov}(i, j))_{1 \leq i, j, \leq n} \\
& \operatorname{cov}(i, j)=\frac{\sum(i-\bar{i})(j-\bar{j})}{n-1}
\end{aligned}
$$

- Calculation of eigenvalues and eigenvectors of $\mathrm{M}$.

- Index of eigenvectors in increasing order of eigenvalues associated.

- Choice the of $m$ more representative eigenvalues ("greatest") and eigenvectors associated to form the characteristic vector $(\mathrm{CV})$ :

$$
F=\left(f_{1} f_{2} f_{3} \ldots f_{m}\right)
$$

1141Projection of the sample in a new sub-space vector $(n-m)$ dimensional, through the expression: 


$$
D F=V C^{T} \times D A^{T}
$$

Thus, a capsule to be compared and possibly identified in the image must, firstly, be projected by equation 3 in that new sub-space. It is then calculated the Euclidean distance of that image with the others that make up the database images. Having established a minimum threshold for acceptance of identification, the image is recognized.

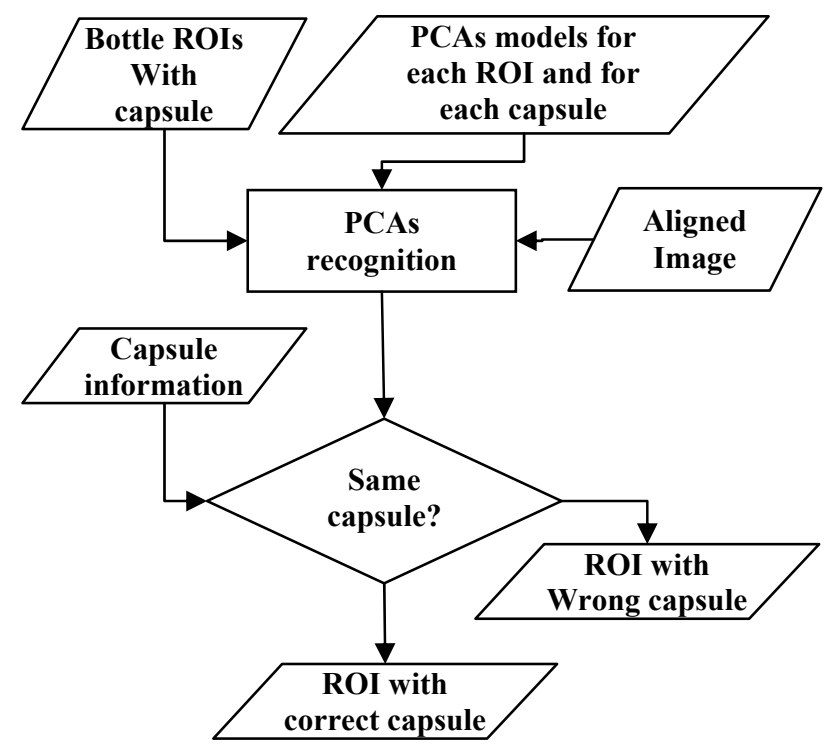

Fig. 12. Algorithm for the recognition of the capsule.

\section{Results and Discussion}

The system is currently under validation and fine tuning in the assembly line. The discussion and the results presented are based on 26000 images of crates of beer distributed by the various items according to the rate of production. This takes $60 \%$ of red crates and $40 \%$ of yellow crates. Concerning the type of beer it represents $45 \%$ of Superbock beer, $30 \%$ of Cristal beer and $25 \%$ of Stout. The analysis of results of the item recognition capsule was carried out in 624.000 capsules. Figures 13, 14 and 15 show the reference image to the uniform illumination, an image acquired on 2 types of crates (yellow and red) and its result after the calibration of the lighting.

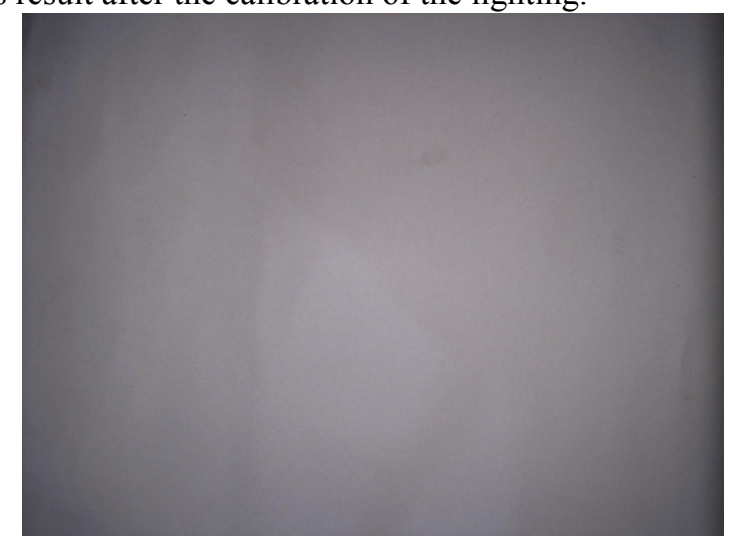

Fig. 13. Image of a white material used to calibrate the lighting in the crate images.
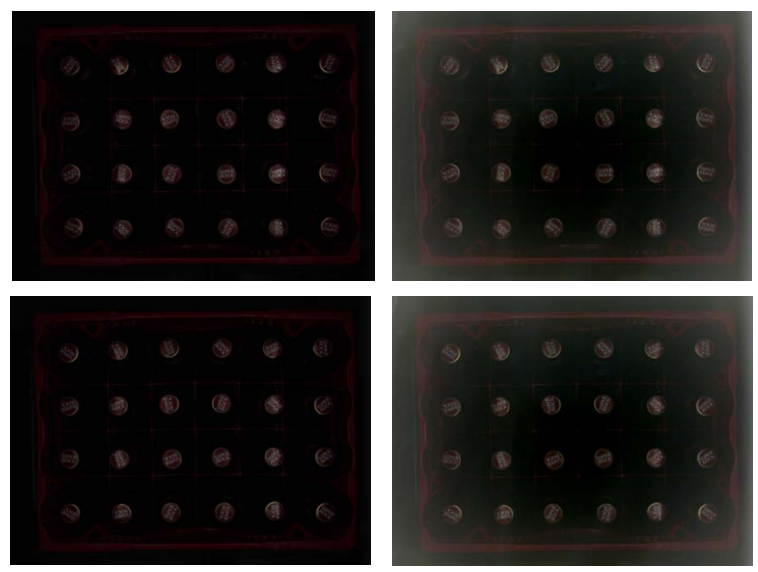

Fig. 14. Images acquired from two red crates (left) and the respective lightning calibration (right).
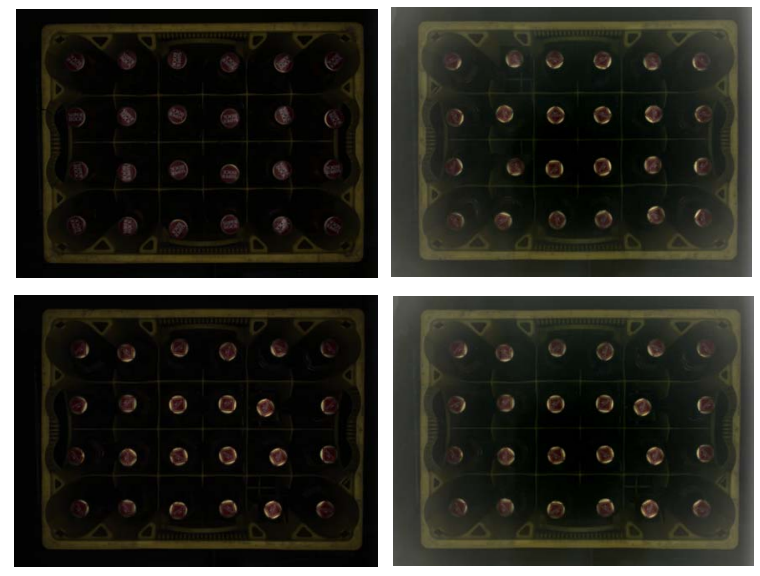

Fig. 15. Images acquired from two yellow crates (left) and the respective lightning calibration (right).

The system was originally developed and parameterized in laboratory. At the stage of system integration in the production line, the parameters of the different algorithms, thresholds binarization and area thresholds, were manually adjusted. The system was parameterized in an optimistic way, allowing the existence of false rejections so that all defects were detected. The false rejection presented for the whole application is $0.74 \%$, regardless of the type of crate and bottle. Specifically, $0.43 \%$ for the items related with the crate checking, $0.14 \%$ for the verification of the bottle and segmentation of the capsule and $0.17 \%$ for the recognition of the capsule. The item to inspect that required greater analysis and parameterization was the recognition of the capsule. As mentioned previously the bottles are not presented to the system with the pattern of the capsules always in the same orientation. The first consequence was the need to introduce models of capsules in which PCAs could reflect this variability of orientations. The perspective effect and specular reflections, present with greater intensity in the periphery of the crate, led to the creation of a set of pattern models for each position of the bottle in the crate and for each type of capsule. In the end, the system has a database of models containing between 16 and 24 models of patterns by crate position and depending on the type of capsule. 
In the laboratory, the image plane GREEN was used for the PCAs. Concerning the recognition of the capsule in industrial environment, the image plane that showed better results was the S of the HSL color space. This was due to the existence of specular reflections and lighting nonuniformity of the color. For each capsule were evaluated the 5 most representative patterns of the capsule in question. The system classifies the capsule by corresponding to the pattern whose number of occurrences is greater than or equal to 3 . Currently the system has a false rejection of the order of $0.17 \%$ distributed as follows by the different types of patterns of the capsules: $30 \%$ for the bottle of beer Superbock, $60 \%$ for the bottle of beer Cristal and $10 \%$ for the bottle of beer Stout. It was intended that the system breaks down 2 crates per second. The currently processing time is about $400 \mathrm{~ms}$ so the system meets the initial specifications.

\section{Conclusions}

A computer vision system was developed to inspect different items on the final stage of a filling bottles production line. Due to the structure of the assemblage line a dedicated industrial setup was specified and a lab prototype was developed for testing performance. The limited physical space available to install the vision system imposed some constrains to the acquired image.

For the different type of items to inspect - crate color; presence of broken crate; presence of bottle; capsule recognition - specific image processing and analysis algorithms were developed. For each defect a set of setup parameters were identified to allow a higher flexibility of the system. The system is already installed on the assemblage line and the tests performed indicate that the approach has confidence for full industrialization. The false rejection of the overall system is of the order of $0.74 \%$, statistics obtained with 26000 images of crates, acquired in the industrial environment.

\section{References}

[1] Rafael C. Gonzalez, Richard E. Woods, "Digital Image Processing", Prentice Hall, 1992.

[2] John C. Russ, "The Image Processing Handbook", 2dh edition, CRC Press, 1995.

[3] Paul Suetens, Pascal Fua, Andrew J. Hanson "Computational Strategies for Object Recognition", ACM Computing Surveys, Vol 24, No 1, March 1992.

[4] OpenCV, "Open Source Computer Vision Library", in http://netaro.ddo.jp/techinfo/OpenCV/opencv094man/index.htm, (03-09-2009)

[5] Dana H. Ballard, Christopher M. Brown, Computer vision, New Jersey: Prentice Hall, 1982.

[6] Donald Hearn, M. Pauline Baker, Computer Graphics Prentice Hall International, pp. 302-205, 1986, ISBN 0-13165598-1. HSV

[7] 6 R. Kramer, Chemometric Techniques for Quantitative Analysis, (1998) Marcel-Dekker, ISBN 0-8247-0198-4.

[8] J. Canny. A Computational Approach to Edge Detection, IEEE Trans. on Pattern Analysis and Machine Intelligence, 8(6), pp. 679-698 (1986).

[9] M. Turk, A. Pentland, Eigenfaces for Recognition, Journal of Cognitive Neurosicence, Vol. 3, No. 1, 1991, pp. 71-86
[10]M.A. Turk, A.P. Pentland, Face Recognition Using Eigenfaces, Proceedings of the IEEE Conference on Computer Vision and Pattern Recognition, 3-6 June 1991, Maui, Hawaii, USA, pp. 586-591

[11]H. Moon, P.J. Phillips, Computational and Performance aspects of PCA-based Face Recognition Algorithms, Perception, Vol. 30, 2001, pp. 303-321. 